Flórez Gutiérrez, A., Ome Moreno, P.A. \& Castellanos Adarme, M.E. (2020). Evidencia del desarrollo del pensamiento de orden superior en profesores universitarios, a partir de pruebas formuladas según la taxonomía SOLO. Revista Electrónica Interuniversitaria de Formación del Profesorado, 23(3), 187-200.

DOI: https://doi.org/10.6018/reifop.434961

\title{
Evidencia del desarrollo del pensamiento de orden superior en profesores universitarios, a partir de pruebas formuladas según la taxonomía SOLO
}

\author{
Alfredo Flórez Gutiérrez ${ }^{(1)}$, Paola Andrea Ome Moreno ${ }^{(2)}$, Mariel Evelyng Castellanos \\ Adarme $^{(3)}$ \\ ${ }^{(1)}$ Universidad Simón Bolívar, ${ }^{(2)}$ Universidad Metropolitana de Educación, Ciencia y \\ Tecnología (UMECIT), ${ }^{(3)}$ Universidad Nacional Experimental de la Fuerza Armada.
}

\section{Resumen}

La propuesta investigativa buscó evidenciar la presencia de pensamiento de orden superior (POS), en docentes universitarios a partir de la aplicación de un instrumento de diagnóstico enfocado en las competencias y estructurado mediante la taxonomía SOLO (Structure of Observed Learning Outcome) y la teoría de respuesta al ítem (TRI), propuesta en el modelo Rasch. El instrumento fue elaborado por docentes de la Corporación Universitaria del Caribe (CECAR), en el marco de un proceso de fortalecimiento de la calidad educativa denominada "Enseñar a Pensar". Los resultados demuestran la efectividad del instrumento para determinar la relación directa entre la habilidad de respuesta frente al ítem, la identificación de niveles de formación según la taxonomía SOLO y la presencia de POS. Sin embargo, el diagnóstico no fue muy satisfactorio, ya que solo el $4.3 \%$ y el $6.6 \%$ de la población docente respondió las preguntas del nivel abstracto ampliado, sobre las competencias de lectura crítica y razonamiento cuantitativo, respectivamente.

\section{Palabras clave}

Pensamiento de orden superior; instrumentos de evaluación; enfoque de competencias; taxonomía SOLO. 


\title{
Evidence of the development of higher order thinking in university professors, from test formulated according to SOLO taxonomy
}

\begin{abstract}
This paper aims to show the presence of higher order thinking, in university teachers through the application of a diagnostic instrument focus on competencies and structured by using the SOLO taxonomy (Structure of Observed Learning Outcome) and the item response theory (IRT), proposed in the Rasch model. The instrument was developed by teachers from CECAR, within the framework of a process of strengthening educational quality called "Teaching to Think". The results demonstrate the effectiveness of the instrument to determine a direct relationship between the response ability against the item, the identification of training levels according to SOLO taxonomy and the presence of higher order thinking. However, the diagnosis was not very satisfactory, due to only $4.3 \%$ y el $6.6 \%$ of the teachers answered the questions of the extended abstract level, about the critical reading skills and quantitative reasoning respectively.
\end{abstract}

\section{Key words}

Higher order thinking; evaluation instruments; competency approach; SOLO taxonomy.

\section{Introducción}

La propuesta investigativa se enmarca dentro de una estrategia para el mejoramiento de la calidad en una institución universitaria de Colombia de carácter privado, que atiende población estudiantil proveniente mayoritariamente en los estratos 1, 2 y 3. La estrategia denominada "Ruta Enseñar a Pensar", tiene como objetivo principal desarrollar el pensamiento de orden superior (POS) en los estudiantes universitarios. Para tal fin, el proyecto se inicia con el trabajo participativo de los profesores quienes colaboran en el diseño del instrumento y en la realización del diagnóstico, a partir de un método multimodal, con el cual se busca evidenciar el grado de desarrollo de POS en los docentes, para que después estos propicien prácticas educativas que favorezcan el aprender a pensar y actuar en los educandos. Es así como la aplicación y validación de un instrumento diseñado con el enfoque de competencias y estructurado mediante la taxonomía SOLO, fue una de las técnicas de diagnóstico, sobre cuyos resultados se centran el propósito de este artículo.

En este sentido, el objetivo de la investigación está relacionado con determinar el nivel de formación de pensamiento de orden superior, en los docentes, a partir de los resultados del instrumento de evaluación estructurado. Construir internamente y aplicar instrumentos de evaluación validados a los docentes, asignar calificaciones en una única escala en forma periódica y sistemática, para conocer quiénes han desarrollado POS, constituyen entonces los objetivos específicos asociados a la investigación.

\section{Origen e importancia del desarrollo del POS en el contexto universitario}

El concepto de POS se deriva de los estudios de la psicología, asociada en cierta forma a la complejidad biológica y neuronal requerida para el desarrollo de lo que se denominarán funciones cognitivas; se refiere, entonces, a capacidades naturales presentes en todos los humanos y que marcan la diferencia con los otros animales. De esta manera, si se tiene en cuenta el enfoque naturalista manejado por Piaget (2009), se describe como un proceso de 
autorregulación desarrollado por estructuras de tipo elevadas y complejas orientadas a buscar el equilibrio entre las acciones en doble vía entre el organismo y el medio. Desde otra perspectiva, Vigotsky $(1978,2000)$ desarrolló un enfoque genético evolutivo, en el que se plantea que las funciones cognitivas de orden superior se generan gracias a la interacción entre lo biológico y lo histórico-cultural. Luria (2000) complementa los aportes de Vigotsky al establecer que dichas funciones son desarrolladas por centros combinatorios en todo el cerebro, cuya ubicación no es estática y cuya respuesta no siempre involucra la misma ruta. Vigotsky (2000) y Luria (2000) describen, entonces, las funciones cognitivas como procesos complejos, autorregulados conscientes y voluntarios. Establecen, también, que las funciones cognitivas superiores involucran una alta gama de ejercicios, entre los que se encuentran las actividades intelectuales. Estas actividades intelectuales son las que en el campo educativo han sido denominadas POS.

Cabe anotar que los estudios de Piaget (2009) y Vigotsky (1978) dieron origen al POS en el ámbito educativo. A estos se les suma el trabajo de Gardner (2016), llamado Proyecto Zero de Harvard, el cual fue considerado como una de las propuestas educativas más disruptivas de los últimos tiempos y que recobra su importancia al ubicar la búsqueda de comprensión como el eje central del aprendizaje (Fernández y Pinzón, 2017).

A partir de la propuesta anterior, se derivan diversos y significativos aportes a la educación, como lo son: la teoría de las inteligencias múltiples de Gardner (1995), la pedagogía de la comprensión de Perkins (1995), las rutinas de pensamiento de Costa y Kallick (2005), el aprendizaje basado en el pensamiento de Swartz, Costa, Beyer, Reagan y Kallick (2008) y los trabajos basados en el desarrollo de pensamiento de orden superior (HOT) o de habilidades de pensamiento de orden superior (HOTS) propuestos por autores como Resnick (1987), Lewis y Smith (1993) y King, Goodson y Rohani (1998).

El POS no posee una definición única y sencilla, pero sus exponentes concuerdan en describirlo como aquel pensamiento esforzado, que tiende a ser complejo y no algorítmico, que solo se genera cuando se asocia la información nueva con la almacenada para generar respuesta a diversas situaciones o encontrar un propósito, lo que implica la aplicación de criterios múltiples, a veces contradictorios entre sí. El POS admite la incertidumbre y requiere de la autorregulación de los procesos de pensamiento. Asimismo, el POS depende de la capacidad para aplicar y reorganizar el conocimiento, por parte de los individuos, quienes también están muy influenciados por el contexto de la situación del pensamiento. Por consiguiente, comprende también el desarrollo de la metacognición y la metaestrategia como acción que connota conciencia, control y aplicación de pasos para pensar y actuar (Resnick, 1987; Lewis y Smith, 1993; King et al., 1998).

El POS ha logrado alcanzar, como categoría de aprendizaje, una concepción holística e incluyente que sobrepasa las intenciones del pensamiento crítico fundamentada en el hecho de considerar procesos reflexivos y favorecer la solución de problemas a partir de apuestas innovadoras, creativas y complejas. Estas apuestas se logran cuando se imparten procesos de enseñanza-aprendizaje que favorecen el desarrollo de los procesos mentales o las habilidades de pensamiento necesarias para tal fin.

El trabajo educativo sobre POS deriva también en planteamientos de clasificación sobre los procesos de pensamiento y los niveles de aprendizaje, como los desarrollados por Bloom (1956), quien considera a las habilidades y capacidades intelectuales como modos organizados de operación y técnicas utilizadas en la manipulación de materiales y problemas; clasifica las habilidades de pensamiento en inferior (conocimiento, comprensión) y superior, (aplicación, análisis, síntesis y evaluación); Anderson y Krathwohl (2001), quienes realizaron ajustes a la taxonomía de Bloom, incorporando la habilidad de crear, como la mayor categoría 
del pensamiento superior; y Biggs y Collis (2005), quienes proponen una nueva organización al plantear la Taxonomía SOLO: dos niveles cuantitativos en los que se concentra la atención en reconocer elementos de un concepto, que se hallan en un contexto; y dos niveles cualitativos, que asocian conceptos y proyectan generalizaciones más allá del contexto dado; estos últimos niveles comprenden funciones cognitivas consideradas de orden superior.

Por último, el POS como tendencia educativa institucionalizada comienza a ganar fuerza en el contexto global, por lo que vale la pena destacar su emergencia en países asiáticos (Malasia e Israel) como política educativa generalizada (Chun y Abdullah, 2019; Seif, 2019).

\section{La taxonomía SOLO y su pertinencia como evidencia del POS}

Esta taxonomía denominada Estructura del Resultado Observado del Aprendizaje, por sus siglas en inglés conocida como (SOLO), según lo refieren Hattie y Brown (2004), fue desarrollada entre los años 1970 y 1980 por los académicos australianos John Biggs y Kevin Collis, y se enfoca en la clasificación de la actividad mental por atributos de cantidad y calidad de las actividades requeridas por los estudiantes o por los productos observables del trabajo que ellos desarrollan.

El enfoque de la taxonomía va hacia el estudio de los resultados generados por los estudiantes en el manejo de distintos cuerpos de contenidos, en este sentido, Biggs (2005), señala que mediante ella se pueden constatar los niveles progresivos y de complejidad estructural en que los estudiantes aprenden, situación que logra evidenciarse mediante la compresión de sus respuestas, productos o resultados generados en atención a una actividad de evaluación. Desde esta perspectiva, se afirma la ocurrencia de dos cambios significativos: uno de carácter cuantitativo que tiene lugar cuando el participante presenta detalles sobre las respuestas entregadas y otro de carácter cualitativo, que se genera cuando demuestra una respuesta o producto estructurado y argumentado que trasciende hacia la comprensión y creación de conocimientos.

Tal como lo refiere Biggs (2005), existen cinco niveles dentro de la taxonomía, en los cuales pueden ubicarse los estudiantes partiendo de los enunciados o detalles, los argumentos y la comprensión que muestran al momento de generar una respuesta o producto ante una evaluación. El primer nivel se conoce como preesctructural: En este se encuentran aquellas respuestas con enunciados erróneos, vacilantes, apoyados en tautologías y de escaso o ningún nivel de comprensión y complejidad respecto de la información abordada. El segundo nivel es el uniestructural: Recoge aquellos enunciados o productos que solo abordan parte de los requerido en una actividad de evaluación. Las respuestas pueden usar terminologías, pero no precisan claridad y coherencia de lo expresado. El tercer nivel es el multiestructural: Se ubican aquí aquellas expresiones que detallan, en mayor o menor grado, los aspectos requeridos en una actividad de evaluación, no obstante, su estructura, coherencia y concreción dista de la verdadera comprensión del foco de aprendizaje. En estos tres niveles se afirma que solo ocurren cambios cuantitativos de aprendizaje; es decir, que existe un mayor manejo de contenidos y acciones. El cuarto nivel es el relacional: En este se presentan aquellas respuestas o productos que poseen un grado de comprensión que supera la mera descripción retórica y manipulación terminológica de datos y hechos, pues abordan los aspectos centrales e inherentes al foco de aprendizaje. Finalmente, el quinto nivel es el abstracto ampliado: Aquí las afirmaciones, respuestas o productos entregados van más allá de lo requerido, al punto que prevalece la coherencia, concreción y la abstracción al evocar conocimientos previos y relacionarlos con la información o contenidos recibidos, y ofrecer argumentos e ideas que evidencian su dominio y aplicación en otros contextos. Desde estas dos últimas etapas, se afirma que suceden cambios cualitativos del aprendizaje, los cuales 
favorecen el pensamiento crítico, reflexivo, coherente y constructivo para apalancar el desarrollo de POS.

Desde esta premisa y en concordancia con Arat (2006), será necesaria la articulación constructiva de todos los factores anteriores para hacer lo siguiente: crear situaciones de aprendizaje que partan desde lo concreto a lo abstracto y cuya intencionalidad se enfoque en diversificar los medios y modos de socializar los cuerpos de contenidos; proponer acciones, retos y desafíos que despierten el interés y la motivación de los estudiantes; e implementar mecanismos de vinculación con escenarios reales donde se intervengan situaciones (simples - complejas) que propicien el establecimiento de criterios, la construcción de significados y nuevas estructuras de orden, la toma de decisiones, reflexión, el manejo de información, la investigación, y la perspectiva sistémica y metacognición inherentes a la presencia de POS. En la tabla 1 se muestra la relación de niveles de taxonomía SOLO con acciones implícitas de POS.

Tabla 1.

Relación Taxonomía SOLO con acciones en el POS.

\begin{tabular}{lll}
\hline $\begin{array}{c}\text { Niveles taxonómicos } \\
\text { según Biggs }\end{array}$ & \multicolumn{1}{c}{$\begin{array}{c}\text { Verbos implícitos en objetivos } \\
\text { curriculares según Biggs }\end{array}$} & Acciones que reflejan POS* \\
\hline Relacional & Comparar Contrastar. & Relacionar información vieja \\
& Explicar causas, & con la nueva. \\
& Analizar, & Reorganizar el conocimiento. \\
& Relacionar, & Generar respuesta a diversas \\
& Aplicar & situaciones. \\
& Teorizar & Aplicar conocimiento. \\
& Generalizar & Encontrar un propósito. \\
& Formular hipótesis & Criticidad. \\
& Reflexionar & Solucionar problemas. \\
& & Autorregulación. \\
& & Creatividad. \\
\hline
\end{tabular}

*Acciones implícitas extraídas de las definiciones provenientes de Resnick (1987), Lewis y Smith (1993) y King et al. (1998).

Fuente: elaboración propia

Las habilidades u operaciones implícitas en el POS, según Shardakov (1985), se desarrollan de las más sencillas a las más complejas, siendo necesarias las primeras para la generación de las segundas. De esta manera, atendiendo a lo planteado por Unigarro (2018), las habilidades específicas implícitas frente a las competencias de lectura crítica y razonamiento cuantitativo en los niveles relacional y abstracto ampliado se pueden apreciar en la tabla 2.

Tabla 2.

Comparación niveles de Taxonomía SOLO con habilidades del POS.

\begin{tabular}{|c|c|c|}
\hline Nivel & Lectura crítica & Razonamiento cuantitativo \\
\hline Relacional & $\begin{array}{l}\text { Reconstrucción de la coherencia global del } \\
\text { texto a partir de la identificación de la } \\
\text { unidad temática y de la coherencia local, de } \\
\text { la jerarquización de ideas y del } \\
\text { establecimiento de relaciones entre las } \\
\text { partes. Genera procedimientos de lectura } \\
\text { inferencial (análisis, integración, } \\
\text { exposición de causas, discusión, }\end{array}$ & $\begin{array}{l}\text { Manejo eficaz de las estrategias de } \\
\text { cálculo, representación de situaciones } \\
\text { a partir del trabajo con modelos } \\
\text { matemáticos y sus condiciones o } \\
\text { supuestos; } \\
\text { representaciones matemáticas con } \\
\text { situaciones reales, explicación con } \\
\text { fluidez de razonamientos e }\end{array}$ \\
\hline
\end{tabular}




\begin{tabular}{|c|c|c|}
\hline & $\begin{array}{l}\text { explicación, categorización, enlazamiento } \\
\text { (Unigarro, 2018, p. 309) }\end{array}$ & $\begin{array}{l}\text { interpretaciones (Unigarro, 2018, p. } \\
\text { 309). }\end{array}$ \\
\hline $\begin{array}{l}\text { Abstracto } \\
\text { Ampliado }\end{array}$ & $\begin{array}{l}\text { Mayor precisión y detalle en los procesos } \\
\text { de comprensión, inferencia, comparación y } \\
\text { contraste; capacidad integradora de } \\
\text { información a partir del análisis de diversos } \\
\text { textos; generación de significaciones con } \\
\text { posturas críticas. Desarrolla lectura crítica: } \\
\text { generalización, proposición de hipótesis y } \\
\text { presunciones, establecer inferencias, } \\
\text { construcción de criterios fundamentados, } \\
\text { reflexionar, deducción lógica y teorización } \\
\text { (Unigarro, 2018, p. 309). }\end{array}$ & $\begin{array}{l}\text { Resultados rápidos y precisos a partir } \\
\text { de estrategias de cálculo no } \\
\text { convencionales, representación de la } \\
\text { vida real por medio de la creación de } \\
\text { modelos matemáticos; desarrollar } \\
\text { generalizaciones a partir del análisis de } \\
\text { varios documentos; aplicación del } \\
\text { conocimiento matemático al } \\
\text { desarrollo de hipótesis, pronósticos, } \\
\text { predicciones e inferencias (Unigarro, } \\
\text { 2018, p. 309). }\end{array}$ \\
\hline
\end{tabular}

Fuente: elaboración propia

En síntesis, los niveles cualitativos de la taxonomía SOLO asocian los procesos de aprendizaje con la adquisición de POS, por lo que en los resultados de la aplicación del instrumento a los profesores se buscó evidenciar el porcentaje de los que llegaron a desarrollar operaciones que les permitieran ubicar sus respuesta en los niveles relacional y abstracto ampliado, ya que estos son los niveles que representan un proceso formativo que trasciende la memoria y se ubica en el plano de lo propositivo, aplicativo y creativo.

\section{Metodología}

\section{Participantes}

Las personas que participaron en el estudio constituyeron una muestra de 231 docentes de pregrado, los cuales fueron seleccionados de forma deliberada atendiendo a criterios que los ubican como docentes: a) con intención voluntaria de participación; b) adscritos a los distintos programas académicos de pregrado (modalidad presencial); c) profesionales con grado académico de maestría o doctorado; d) que ejercen funciones de investigación e) sin discriminación de género y f) con antigüedad laboral mayor o igual a un año.

\section{Diseño del estudio}

En atención al propósito de la investigación se siguió un diseño descriptivo y de campo para tomar registro, hacer análisis e interpretar la realidad observada que en este caso son los niveles competenciales en lectura crítica y razonamiento cuantitativo que tienen los docentes de los programas académicos de pregrado (modalidad presencial) de CECAR. En consecuencia, con el enfoque cuantitativo de este momento del proceso, se desarrolló un estudio no experimental, transeccional, pues no se enfocó en la manipulación de variables, sino en la ejecución de una medición a través de la cual se recopilaron datos para establecer descripciones sobre la realidad investigada.

Su diseño se estructuró en una fase de revisión sistemática de literatura, dirigida a la consulta, clasificación y recolección de información que facilitó el posicionamiento teórico conceptual relacionado con el POS, la taxonomía SOLO y las implicaciones que estos conllevan para la interpretación de los resultados. Seguidamente, se ejecutó una fase de construcción por parte de los cuerpos colegiados de profesores y, luego, una fase de campo, mediante la cual se planificaron las actividades para la aplicación del instrumento, la 
estimación de los recursos y la aplicación de la medición. Finalmente, se procedió con una fase de análisis y contrastación de resultados.

\section{Instrumento de recogida de datos}

El cuestionario utilizado fue construido por docentes de la misma institución (CECAR), con la intención de diagnosticar las competencias y habilidades implícitas y requeridas para la formación de sus estudiantes desde sus contextos disciplinares. No obstante, después se decidió aplicar este instrumento en los maestros bajo la premisa de poder precisar si ellos alcanzaban los niveles óptimos en las capacidades transversales evaluadas, como garantía de la adecuada aplicación y desarrollo de POS en los discentes.

Para construir el instrumento se siguió el procedimiento de calibración, el cual fue elaborado por Bogoya, Barragán, Contento y Ocaña (2014), en atención de la aplicabilidad de la teoría de respuesta al ítem (TRI). Este procedimiento abarcó las siguientes fases: a) la conformación de la colegiatura de docentes que se encargó de establecer el marco teórico y metodológico de los constructos e indicadores medidos a través del instrumento; b) el ensamblaje y aplicación, mediante el cual se integraron los contextos, escenarios, las características de los participantes, relaciones de interacción, los campos contextuales y la contrastación teórica para redactar los ítems en función de identificar la consolidación de las competencias de lectura crítica y razonamiento cuantitativo según los niveles taxonómicos de SOLO; y c) la aplicación de la prueba piloto para concretar su validez y confiabilidad.

En la construcción del instrumento se asumió la integración de bloques contextuales (local, social y global), a partir de los cuales se elaboraron cinco ítems ajustados a los niveles referidos en la taxonomía SOLO (uniestructural [1], multiestructural [1], relacional [2] y nivel abstracto [1]), para cada una de las dos competencias que se iban a examinar. Esto produjo un total de 60 ítems con cuatro opciones de respuestas de las cuales solo una es correcta o válida, además de los rangos de respuestas según los niveles de medición taxonómica SOLO.

Por medio de la validación estadística y retroalimentación, se hizo un análisis riguroso de la calidad del instrumento propuesto y su respectiva prueba piloto, para evidenciar el nivel de dominio de las competencias de razonamiento cuantitativo y lectura crítica presentes en los docentes de la institución. La aplicación de TRI se apoyó en la integración de un parámetro (dificultad) en el modelo de Rasch (como se citó en Baker, 2001), con la intención de examinar cada ítem y evidenciar su validez y la del instrumento para establecer parámetros de caracterización de los sujetos según los niveles de taxonomía SOLO.

La validez y confiabilidad del instrumento se obtuvo mediante el análisis de los datos recopilados en la prueba piloto. A partir de allí, se establecieron valores para los parámetros de dificultad, error de estimación, correlación ítem-prueba, ajuste próximo y lejano, y discriminación de cada uno de los ítems. Con respecto al instrumento, se procedió a calcular su confiabilidad, consistencia interna (Alfa de Cronbach: igual o mayor 0.7) y dimensionalidad a través de la relación entre el porcentaje de varianza explicada por las medidas y el porcentaje de varianza que explica el contraste con los componentes del instrumento. Todo lo anterior permitió obtener la validez de constructo. El producto obtenido en esta etapa se compone de un rango de valores satisfactorios para contrastar los parámetros de los ítems y del instrumento, además de la escala que define el grado de dificulta de los ítems.

\section{Procedimiento de análisis}

El procesamiento de datos se apoyó en el uso de herramientas informáticas WINSTEPS 3.73, en conjunto con el software ConQuest. Además, se ejecutó en tres etapas: la primera denominada precalibración, que se enfocó en identificar y extraer aquellos registros con patrones de respuestas anómalas (omisión, valores negativos en la correlación o que no 
cumplen con el criterio según el modelo fijado), lo que se traduce en una mayor cantidad de información depurada y una mayor consistencia interna. La segunda etapa se denominó calibración. Esta centró sus actividades en el tratamiento estadístico de los datos depurados en la etapa previa, con el propósito de extraer los ítems que no lograron satisfacer los criterios establecidos para calcular el valor de los parámetros de las respuestas dadas a cada ítem del instrumento. La tercera etapa fue la calificación. En esta se procedió a asignar un valor de habilidad para cada profesor que contestó el instrumento, valor que se ajustó a los referidos en la escala de complejidad de cada nivel determinado en la etapa previa. Para este caso, los valores se vincularon con los niveles de complejidad progresiva de la taxonomía SOLO. Básicamente, se procedió a medir el desempeño de los docentes en atención a las cadenas de respuestas generadas y su contrastación con los parámetros establecidos en cada ítem del instrumento.

El análisis de los datos obtenidos se apoyó en el tratamiento estadístico descriptivo de cada ítem, respecto de las competencias de lectura crítica y razonamiento cuantitativo, a través del cual se examinaron las respuestas ofrecidas por los docentes ante cada una de las situaciones referidas en los ítems, los contextos, las respuestas y los niveles de complejidad que determinaba la taxonomía SOLO. Este procedimiento de análisis se representó mediante gráficos circulares y de barras acompañados de textos explicativos derivados de la interpretación de los datos.

\section{Análisis y resultados:}

Para la construcción del instrumento, se establecieron dos (2) competencias a evaluar: lectura crítica y razonamiento cuantitativo; las cuales fueron desarrolladas por dos equipos de trabajo conformado por diez (10) docentes, cada uno articulado con una competencia según su proceder disciplinar, tomando en consideración la ruta metodológica dispuesta para el diagnóstico. Durante el diseño del instrumento, se acordó la construcción de cuatro (4) bloques conformados cada uno por tres contextos (local, social y global) y cada uno de ellos con cinco ítems distribuidos y ajustados a los niveles referidos en la taxonomía SOLO (uniestructural [1], multiestructural [1], relacional [2] y nivel abstracto [1]). El instrumento se integró a MOODLE que se encuentra alojado dentro de la plataforma institucional con un tiempo estimado para su respuesta de 120 minutos; y el tiempo de aplicación fue de una semana.

Los constructos evaluados presentaron valores para el coeficiente Alfa de Cronbach, que da cuenta de la consistencia de las respuestas obtenidas, iguales a 0.23 en lectura crítica y 0.36 en razonamiento cuantitativo; mientras que la confiabilidad se ubicó en 0.66 para lectura crítica y 0.73 para razonamiento cuantitativo.

Los resultados obtenidos en la aplicación sobre las competencias en lectura crítica y razonamiento cuantitativo demuestran que solo el $40.7 \%$ y el $33.9 \%$ respectivamente, de la muestra evaluada (231 docentes) se ubican en los dos niveles cualitativos más complejos de la taxonomía SOLO (el relacional y abstracto ampliado). Para efectos del análisis, a excepción del nivel abstracto ampliado, los tres niveles anteriores fueron subdivididos en alto y bajo, diferenciando los que están por debajo y por encima del valor medio de cada nivel. En este sentido, se observa que el mayor porcentaje de la población se ubica en el nivel multiestructural, alto. Mientras que, solo el $4.3 \%$ y el $6.6 \%$ de los profesores, se ubican en el nivel abstracto ampliado en lectura crítica y razonamiento cuantitativo, respectivamente (ver figuras $1,2,3$ y 4 ). 
Llama la atención que el porcentaje de profesores ubicados en el nivel uniestructural sea más alto que los que se ubican en el abstracto ampliado ( $5.5 \%$ en lectura crítica y $8.2 \%$ en razonamiento cuantitativo), lo que se considera un factor neurálgico, dado su estatus de profesionales y sobre todo de educadores, cuya función es contribuir a la óptima formación de otros seres humanos.

\section{Lectura Crítica - \% Profesores}

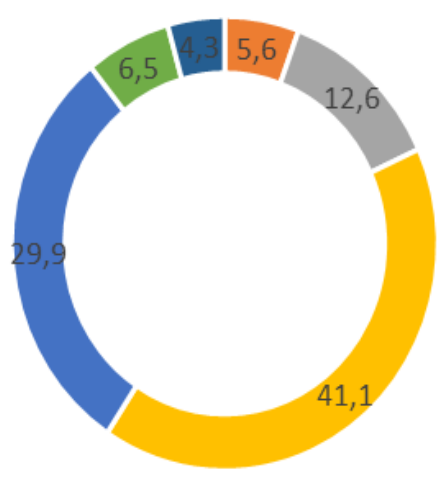

- Bajo uniestructural $\quad$ Uniestructural $\quad$ Multiestructural bajo

- Multiestructural alto - Relacional bajo $\square$ Relacional alto

- Abstracto ampliado

Figura 1. Resultados lectura crítica.

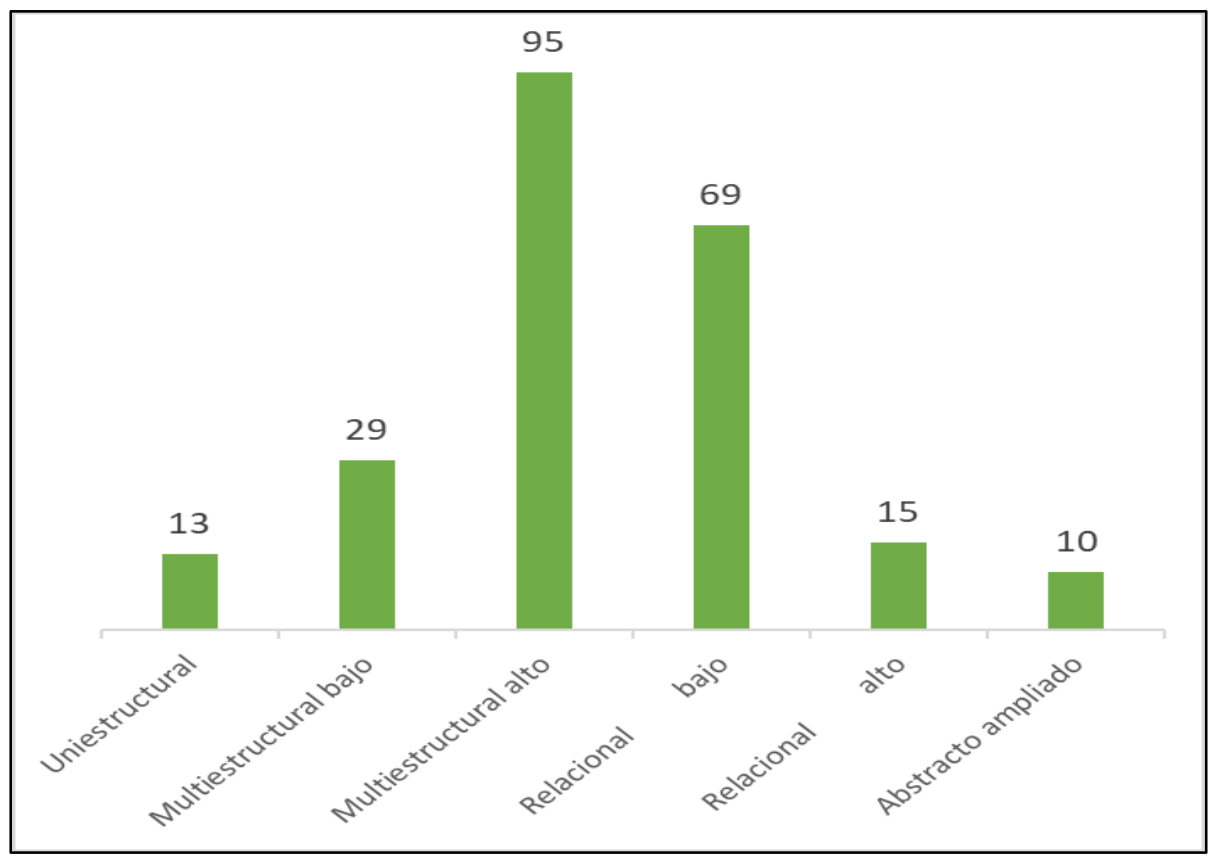

Figura 2. Resultados lectura crítica. 


\section{Razonamiento Cuantitativo - \% Profesores}

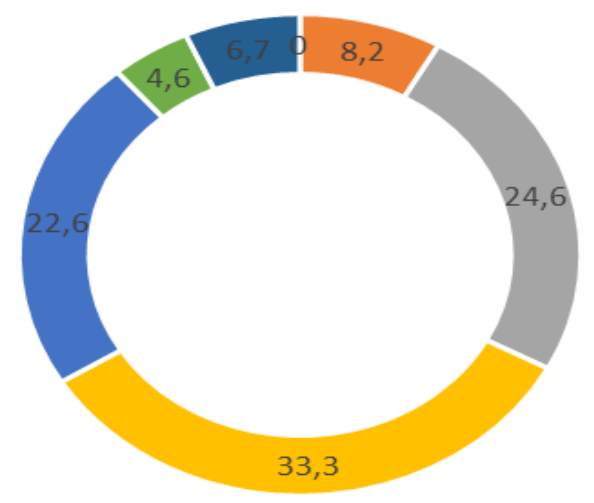

$$
\begin{aligned}
& \text { Bajo uniestructural } \square \text { Uniestructural } \\
& \text { Multiestructural bajo } \square \text { Multiestructural alto } \\
& \text { Relacional bajo } \square \text { Relacional alto }
\end{aligned}
$$

- Abstracto Ampliado

Figura 3. Resultados razonamiento cuantitativo.

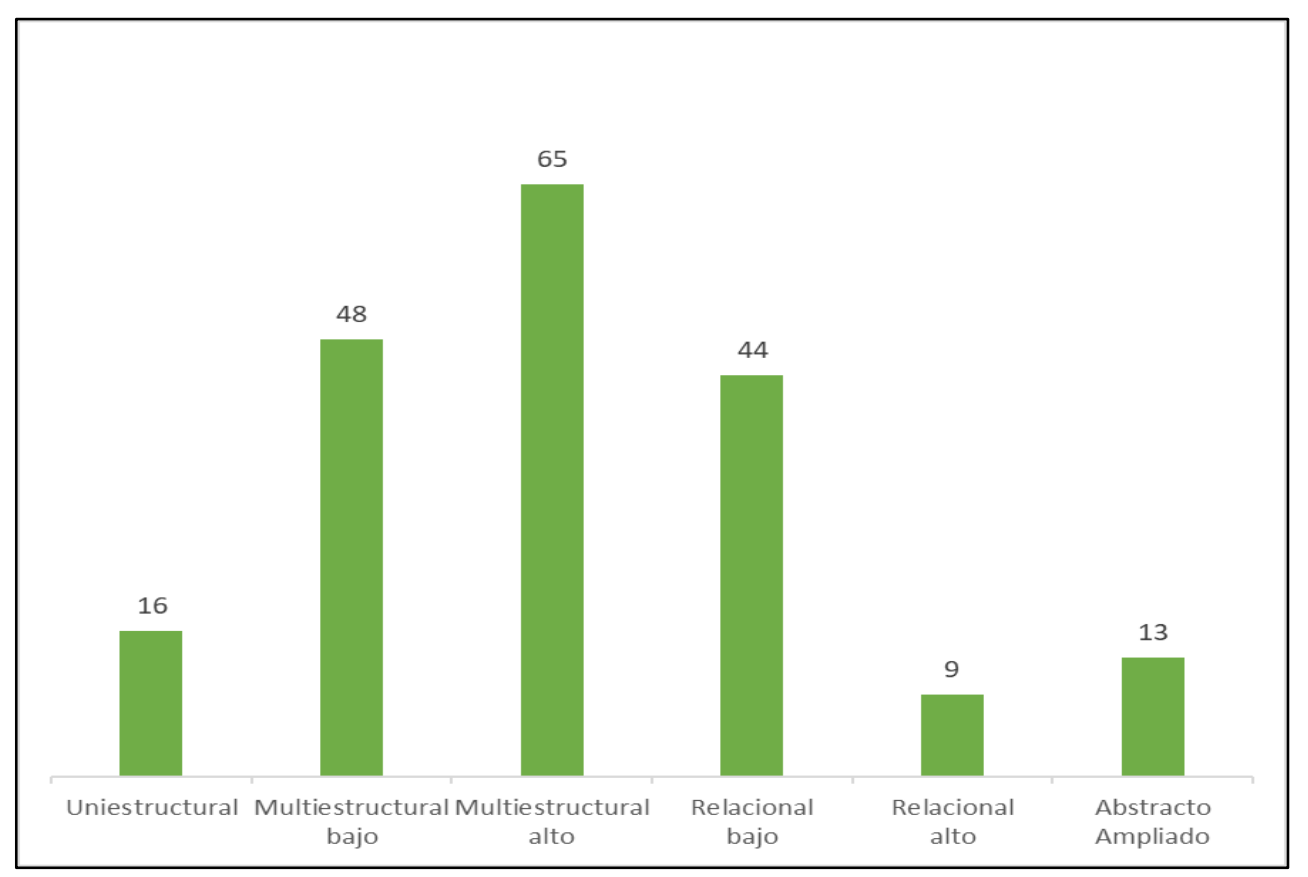

Figura 4. Resultados razonamiento cuantitativo.

En consecuencia, amparado los resultados se puede constatar la validez del instrumento en cuanto al índice de respuesta al ítem y su grado de dificultad, lo que conlleva a que solo los docentes que demuestran un adecuado desarrollo de POS, llegan a resolver las preguntas asociadas a los niveles relacional y abstracto ampliado 
Ahora bien, es importante tener claro que según el modelo de Rash, existe una relación directa entre la complejidad del ítem, con la presencia de mayor o menor habilidad del evaluado (Baker, 2001). En consecuencia, amparado los resultados se puede constatar la validez del instrumento en cuanto al índice de respuesta al ítem y su grado de dificultad, lo que conlleva a que solo los docentes que demuestran un adecuado desarrollo de POS, llegan a resolver las preguntas asociadas a los niveles relacional y abstracto ampliado.

La habilidad se entiende como una capacidad cognitiva compleja que posibilita el desarrollo de operaciones; por consiguiente, como se sustentó anteriormente, si se tienen en cuenta las acciones implícitas en los niveles relacional y abstracto ampliado, el POS solo se evidencia en el $37.3 \%$ de los profesores, sobre las respectivas competencias evaluadas (ver la tabla 3 ).

Tabla 3.

Relación de porcentaje de docentes ubicados en cada nivel de la taxonomía.

\begin{tabular}{lcc}
\hline Niveles & \multicolumn{2}{c}{$\mathbf{N}^{\circ}$ Profesores } \\
\hline $\begin{array}{l}\text { Nivel uniestructural (con menor habilidad } \\
\text { o competencia). }\end{array}$ & $13 / 5.6 \%$ & $\begin{array}{c}\text { Razonamiento } \\
\text { cuantitativo }\end{array}$ \\
\hline Multiestructural & $124 / 53.7 \%$ & $16 / 8.2 \%$ \\
\hline Relacional & $84 / 36.3 \%$ & $113 / 57.9 \%$ \\
\hline $\begin{array}{l}\text { Abstracto ampliado (muy hábiles } \\
\text { competentes) }\end{array}$ & $10 / 4.3 \%$ & $13 / 6.1 \%$ \\
\hline
\end{tabular}

Fuente: elaboración propia

\section{Discusión y conclusiones}

Se evidencia así un problema en la calidad académica en la institución universitaria, asociada al nivel de competencias y habilidades presentes en la mayoría de sus docentes, quienes en un bajo porcentaje llegan a responder preguntas asociadas al nivel abstracto ampliado, lo que implica muy poca presencia de POS en los mismos. De allí que, cobre importancia el proceso desarrollado, ya que se requiere institucionalmente de mecanismos para apropiar la evaluación como un componente más de la cultura académica.

Así pues, se puede considerar un logro la construcción de instrumentos propios de evaluación, los cuales se enmarcan dentro de un proceso de formación de los docentes, lo que contribuye a desarrollar y consolidar de manera consciente, la actuación del docente frente al desarrollo de pensamiento de orden superior y su necesidad de autorregularse.

Ahora bien, los resultados podrían estar indicando una relación directa entre las capacidades del profesor con el modelo educativo desde el que fueron formados, lo que corrobora la fuerte presencia de procesos de memorización, poca asociativa y resolución de problemas. Lo anterior se ratifica, si se compara los resultados de los docentes frente al desempeño de los estudiantes de CECAR en las pruebas nacionales de evaluación de competencias genéricas (Prueba Saber Pro) aplicadas por el Instituto Colombiano para el Fomento de la Educación Superior (ICFES, 2019), que reflejan un posicionamiento muy por debajo del promedio nacional, tal como se aprecia en la figura 6. 
En este sentido, la calidad de la formación de los estudiantes, no se puede mejorar, hasta que los docentes no superen sus propias limitaciones, lo que implica centrar esfuerzos para fortalecer la formación de los docentes.

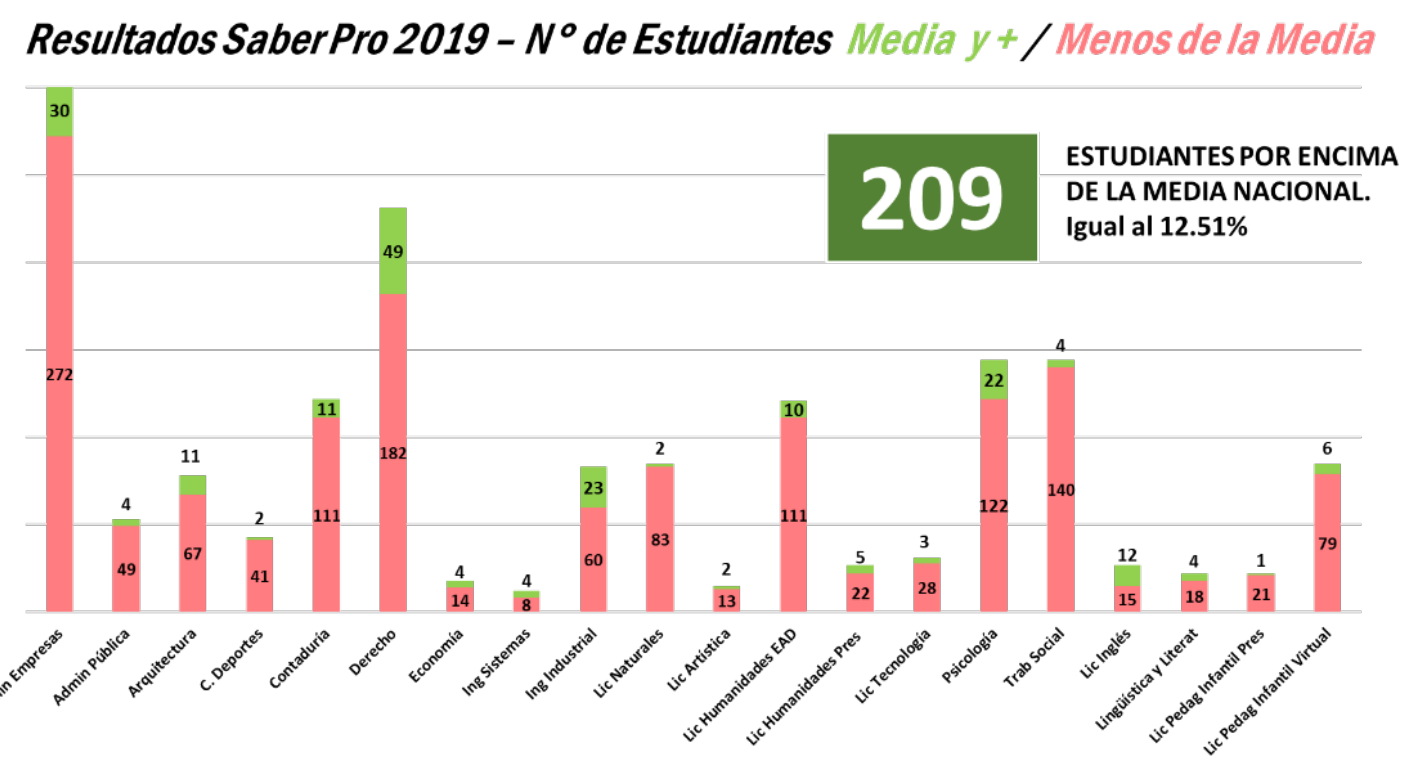

Figura 6. Resultados Saber Pro 2019 -Estudiantes.

Por consiguiente, el bajo desempeño de los docentes en el diagnóstico establecido debe considerarse como punto de partida para desarrollar toda una estrategia integral que favorezca el cambio de paradigma frente a su praxis formativa, ya que implica procesos tanto de autofortalecimiento como de apropiación de metodologías, para trascender el modelo educativo memorístico predominante.

Es así, como se podrían proponer estrategias formativas focalizadas en las necesidades de cada docente, que además de favorecer el fortalecimiento frente al POS, promuevan la aplicación de nuevas didácticas de aula para el desarrollo de las mismas en lo estudiantes.

Con lo anterior, de la fase diagnóstico de la investigación se puede concluir que:

- Se logró construir y validar institucionalmente, un instrumento de evaluación propio, para determinar el grado de desarrollo del POS en los docentes.

- El instrumento estructurado bajo la taxonomía SOLO, es apropiado para determinar POS, toda vez que los niveles de dicha taxonomía relacionan operaciones mentales y habilidades, consideradas como de Pensamiento de Orden Superior.

- El ejercicio de diagnóstico posibilitó establecer institucionalmente un mecanismo de evaluación y medición que será aplicado anualmente a los docentes, con el ánimo de determinar su progreso frente a las capacidades cognitivas requeridas.

- Como curso de acción el ejercicio de diagnóstico consolida la base de la estrategia a desarrollar con los docentes en la Ruta Enseñar a Pensar, lo que se convierte en un mecanismo para evitar el activismo y favorecer la planeación estratégica académica.

Por lo anterior, se considera que el proceso investigativo generó logros significativos para consolidar una verdadera estrategia de seguimiento de la calidad académica del docente en CECAR. 


\section{Referencias}

Anderson, W. I. y Krathwohl, D. R., (Eds.), Airasian, P.W., Cruikshank, K.A., Mayer, R.E., Pintrich, P.R., ... y Wittrock, M.C. (2001). Una taxonomía para el aprendizaje, enseñanza y evaluación: una revisión de la taxonomía de Bloom de objetivos educativos. Boston: Allyn y Bacon.

Anat, Z. (2006). El pensamiento de orden superior en las clases de ciencias: objetivos, medios y resultados de investigación. Enseñanza de las Ciencias: revista de investigación y experiencias didácticas. 24(2), pp. 157-172. Recuperado de https://www.raco.cat/index.php/Ensenanza/article/view/75823/96327

Baker, F. (2001). The basic of item response theory. United States of America: ERIC Clearinghouse on Assessment and Evaluation.

Biggs, J. B., \& Collis, K. F. (1982). Evaluating the quality of learning: The SOLO taxonomy (Structure of the Observed Learning Outcome). New York: Academic Press.

Biggs, J. B. (2005). Calidad del aprendizaje universitario. Madrid: Narcea S.A.

Bloom, B. S. (1956). Taxonomy of educational objectives: The classification of educational goals, handbook 1 cognitive domain. New York: David McKay Co. Inc

Bogoya, D., Barragán S., Contento, M. y Ocaña, A. (2014). Calibración de instrumentos de evaluación - clasificación en matemáticas en la Universidad Jorge Tadeo Lozano. Revista Complutense de Educación. 25(2). 501-519. Recuperado de http://dx.doi.org/10.5209/rev_RCED.2014.v25.n2.41931

Costa, A., \& Kallick B. (2005) Habits of mind. A curriculum for Community High School of Vermont Students. Edited by Arthur L Costa y Bena kallick. Recuperado de https://www.chsvt.org/wdp/Habits_of_Mind_Curriculum_VT_WDP.pdf

Chun, T y Abdullah, M (2019). The teaching of higher order thinking skills (HOTS) in Malaysian schools: Policy and practices. Malaysian Online Journal of Educational Management. , 7(3), $1-18$.

Fernández, A. y Pinzón, G. (2017). ¿De qué debemos desprendernos para cambiar la educación? En A. Forés y E. Subias, Pedagogías emergentes 14 preguntas para el debate (pp. 41-56). Barcelona: Octaedro.

Gardner, H (1995). Inteligencias múltiples. La teoría en la práctica. Barcelona-España: Paidós.

Gardner, H. (2016). El Proyecto Zero de Harvard: una historia personal. Escuela de graduados en Educación de la Universidad de Harvard. Uaricha, 13 (30), 26-52.

Hattie, J.A.C., \& Brown, G.T.L. (2004). Cognitive processes in asTTle: The SOLO taxonomy. asTTle Technical Report \#43, University of Auckland/Ministry of Education.

Instituto Colombiano para el Fomento de la Educación Superior. (2019). Informe de resultados Saber Pro. Recuperado de https://www2.icfes.gov.co/resultados-saber-pro

King, F.J., Goodson, L., \& Rohani, F. (1998). Higher order thinking skills: Definition, teaching strategies and assessment. Publication of the Educational Service Program. Recuperado de https://informationtips.files.wordpress.com/2016/02/higher-orderthinking-skills_.pdf

Lewis, A., \& Smith, D. (1993). Defining higher order thinking: Theory into practice, 32 (3), 131137, doi: 10.1080/00405849309543588. Recuperado de https://www.tandfonline.com/doi/abs/10.1080/00405849309543588 
Luria, A. (2000). Las funciones corticales superiores del hombre. México D.F.: Ediciones Fontamara. México. D.F.

Perkins, D. (1995) La escuela inteligente. Barcelona: Gedisa.

Piaget, J. (2009). La psicología de la inteligencia. Barcelona: Biblioteca de Bolsillo.

Resnick, L. (1987). Education and learning to think. Washington D. C.: National Academy Press.

Seif, A. (2019). Integration of higher order thinking skills into the Arab education system in Israel: A general perspective. Italian Journal of Sociology of Education, 11(3), 304-346.

Shardakov, M. (1985). Desarrollo del pensamiento en el escolar. México D.F.: Grijalbo.

Swartz, R., Costa, A., Beyer, B., Reagan, R. y Kallick, B. (2008). El aprendizaje basado en el pensamiento. Cómo desarrollar en los alumnos las competencias del siglo XXI. Vitoria, España: Ediciones SM.

Unigarro, M. (2018). Creación del sistema institucional de evaluación de competencias de la Universidad Cooperativa de Colombia. En S. Castillo, C. Roldan, D. González, O. Rodríguez y C. Ruano. Evaluación de aula, evaluación estandarizada y emergencia de sistemas de evaluación de aprendizajes (pp. 299-316). Cali: Editorial Bonaventura Programa Editorial Universidad Autónoma de Occidente.

Vigotsky, L. (1978). El desarrollo de los procesos psicológicos superiores. Barcelona: Grijalbo.

Vigotsky, L. (2000). Obras escogidas, III. Madrid: Visor S.A. 